Passive Vector Geoacoustic Inversion in Coastal Areas Using a sequential Unscented Kalman Filter

J. V. Candy, Q. Y. Ren, J. P. Hermand

September 12, 2013

IEEE SYMPOL Conference Cochin, India October 23, 2013 through October 25, 2013 
This document was prepared as an account of work sponsored by an agency of the United States government. Neither the United States government nor Lawrence Livermore National Security, LLC, nor any of their employees makes any warranty, expressed or implied, or assumes any legal liability or responsibility for the accuracy, completeness, or usefulness of any information, apparatus, product, or process disclosed, or represents that its use would not infringe privately owned rights. Reference herein to any specific commercial product, process, or service by trade name, trademark, manufacturer, or otherwise does not necessarily constitute or imply its endorsement, recommendation, or favoring by the United States government or Lawrence Livermore National Security, LLC. The views and opinions of authors expressed herein do not necessarily state or reflect those of the United States government or Lawrence Livermore National Security, LLC, and shall not be used for advertising or product endorsement purposes. 


\title{
Passive vector geoacoustic inversion in coastal areas using a sequential unscented Kalman filter
}

\author{
Qun-yan Ren*, James V. Candy ${ }^{\dagger}$ and Jean-Pierre Hermand* \\ *Acoustics and Environmental hydroacoustics Lab, Université libre de Bruxelles (U.L.B.) \\ av. F. D. Roosevelt 50, CP 165/57, B-1050 Brussels, Belgium \\ †University of California Santa Barbara, California, USA
}

\begin{abstract}
An unscented Kalman filter (UKF) for geoacoustic inversion using scalar and vector sound fields created by a passing ship is discussed in this paper. The continuous sound field emitted by a ship of opportunity is processed by the sequential filtering technique to estimate slowly changing environmental properties along the source range. The inversion problem is solved by the UKF with a random-walk parameter model, which is expected to perform well when dealing with highly nonlinear problems. Synthetic geoacoustic inversions are performed using multi-frequency pressure, vertical particle velocity and waveguide impedance (a ratio between pressure and vertical particle velocity) data for the geoacoustic model of a mud environment offshore at the mouth of the Amazon river in Brazil (CANOGA 12). For the preliminary tests, the sound source is composed of a flat spectrum. Numerical results demonstrate that the sequential filtering technique is capable of estimating the evolution of environmental properties along the source range. In practice, ship data have complex time-varying spectral characteristics that can greatly limit the accuracy of broadband or multi-frequency passive applications. Since the vertical waveguide impedance is independent of the source spectral level, it is preferred for environmental characterization by the sound field generated from a ship of opportunity. Because of this independence property, the vertical waveguide impedance is expected to yield a more reliable inversion than that of pressure or vertical particle velocity field.
\end{abstract}

\section{INTRODUCTION}

The capacity of a vector sensor in simultaneously measuring the scalar and vector sound fields at the same point of a waveguide [1] makes it is possible to use the relationship between them for geoacoustic inversion, which may be independent of source characteristics [2]. The vertical waveguide impedance is a ratio of pressure and vertical particle velocity and demonstrated to be source spectrum independent but highly correlated with environmental properties [3]. Such impedance is emphasized here for geoacoustic inversion using source of opportunity, e.g., surface ship.

Geoacoustic inverse problem is usually high dimensional and non-linear problem and solved by matched-fieldprocessing (MFP) technique [4]-[6] with global or local optimization algorithms [7]-[9], to estimate the range-averaged properties of the waveguide over different scales.

Work supported by Belgian National Fund for Scientific Research (F.R.S.F.N.R.S.), National Council for Scientific and Technological Development (CNPq), Wallonie-Bruxelles International (WBI), and Coordenação de Aperfeiçoamento de Pessoal de Nível Superior (CAPES).
Here, an unscented Kalman filter (UKF) [10] is used to estimate environmental properties by observing the range variations of scalar and vector sound fields created by a moving ship. To take advantage of the continuous ship sound field along range, the inverse problem is solved by a random walk parameter model [11], which formulate the environmental parameters in a recursive state-space form along source range. For each range point, their values are estimated through sequentially filtering ship sound field data.

Synthetic tests for geoacoustic inversion are performed for the geoacoustic model of a mud environment offshore at the mouth of the Amazon river in Brazil (CANOGA 12) [12] using multi-frequency pressure, vertical particle velocity and waveguide impedance data. For the preliminary tests, the sound source is composed of a flat spectrum.

The following of the paper is organized as: Section II briefly introduces the scalar and vector acoustical observables. Sequential unscented Kalman filter is presented in Sec. III. Synthetic geoacoustic inversion tests are given in Sec. IV. Sec. V is the conclusions.

\section{SCALAR AND VECTOR ACOUSTICAL OBSERVABLES}

The received acoustic complex pressure field $P$ and vertical particle velocity $V_{z}$ at ranges $r$ and generated by an omnidirectional point source at depth $z_{0}$ emitting a single tone at angular frequency of $\omega$, received at depth $z$ can be respectively expressed as [13]

$$
P(\omega, r, z)=\pi i S(\omega) \sum_{l} \phi_{l}\left(z_{0}\right) \phi_{l}(z) H_{0}^{(1)}\left(\xi_{l} r\right)
$$

and

$$
\begin{aligned}
V_{z}(\omega, r, z) & =\frac{-1}{i \omega \rho} \frac{\partial}{\partial z} P(\omega, r, z) \\
& =\frac{i S(\omega)}{\omega \rho} \sum_{l} \kappa_{l, z} \phi_{l}\left(z_{0}\right) \phi_{l}^{\prime}(z) H_{0}^{(1)}\left(\xi_{l} r\right)(2)
\end{aligned}
$$

where

- $S(\omega)$ is the source amplitude at $\omega$

- $\rho$ is the medium density

- $\phi_{l}$ and $\xi_{l}$ represent the modal function and eigenvalue for the $l$ th mode

- $\phi_{l}^{\prime}$ is the derivative of $\phi_{l}$ with respect to $z$

- $H_{0}^{(1)}$ is the zero order Hankel function of the first kind 
Table I: Scenario geoacoustic model for the CANOGA 12 experiment area.

\begin{tabular}{l|l|c}
\hline Water column & depth $\left(d_{w}\right)$ & $13.5 \mathrm{~m}$ \\
\hline \multirow{3}{*}{ Sediment } & thickness $(h)$ & $2.0 \mathrm{~m}$ \\
& density $\left(\rho_{\text {sed }}\right)$ & $1.27 \mathrm{~g} / \mathrm{cm}^{3}$ \\
& sound speed $\left(C_{\text {sed }}\right)$ & $1450 \mathrm{~m} / \mathrm{s}$ \\
& attenuation $\left(\alpha_{\text {sed }}\right)$ & $0.2 \mathrm{~dB} / \lambda$ \\
\hline \multirow{3}{*}{ Half space } & density $\left(\rho_{\text {bot }}\right)$ & $1.6 \mathrm{~g} / \mathrm{cm}^{3}$ \\
& sound speed $\left(C_{\text {bot }}\right)$ & $1530 \mathrm{~m} / \mathrm{s}$ \\
& attenuation $\left(\alpha_{\text {bot }}\right)$ & $0.25 \mathrm{~dB} / \lambda$ \\
\hline
\end{tabular}

- $\kappa_{l, z}$ is the vertical wavenumber for the $l$ th mode

For clarity, the dependence term $(\omega, r, z)$ of sound fields is omitted in the following text.

Simply, the vertical waveguide impedance component, being the ratio between $P$ and $V_{z}$ can be written as:

$$
Z_{z}=\frac{P}{V_{z}}=\frac{-i \omega \rho \sum_{l} \phi_{l}\left(z_{0}\right) \phi_{l}(z) H_{0}^{(1)}\left(\xi_{l} r\right)}{\sum_{m}^{M} \kappa_{m, z} \phi_{m}\left(z_{0}\right) \phi_{m}^{\prime}(z) H_{0}^{(1)}\left(\xi_{m} r\right)} .
$$

Due the ratio operation, source term effect $S(\omega)$ presents in Eqs. 1 and 2 is eliminated. Consequently, the $Z_{z}$ is source spectrum independent but its range variation is highly correlated with environmental terms, e.g., $H_{0}^{(1)}\left(\xi_{l} r\right) . Z$ is demonstrated to be more sensitive to geoacoustic parameters than that of $P$ and $V_{z}$, especially for bottom densities [3]. These advantages make $Z_{z}$ a valuable physical variable to be observed for passive environmental inversion.

\section{RANDOM WALK PARAMETER MODEL}

The Kalman filter [14] usually estimates unknown variables by observing a series of noisy measurements in a two-step process: prediction and updation (or correction). It first estimates the current state variables as well as their uncertainties and then used in the second step with new measurement to update the estimates. The filter can operate recursively to produce a statistically optimal estimate of the underlying system state. In other words, this filter can intrinsically run on continuous data using only the current inputs and previously estimation.

In our model, the state vector $\mathbf{m}$ contains environmental parameters, and the measurements $\mathbf{Y}$ are the $Z_{z}$ for several frequencies at different ranges:

$$
\begin{gathered}
\mathbf{m}_{r_{k}}=F\left(\mathbf{m}_{r_{k-1}}\right)+W\left(r_{k-1}\right), \\
\mathbf{Y}\left(r_{k}\right)=J\left(\mathbf{m}_{r_{k-1}}, \mathbf{f}\right)+V\left(r_{k-1}\right),
\end{gathered}
$$

where $\mathbf{f}$ is a vector of frequency bins observed, $W$ and $V$ are zero-mean Gaussian noise terms with noise covariances of $R_{w w}$ and $R_{v v}$, respectively. The model becomes a random model when $F$ is identity in Eq. 4 . The acoustic propagation model is embedded in measurement function $J$, which outputs the $Z_{g}$ at different range for the observed frequencies with environmental properties contained in state vector $\mathbf{m}$.
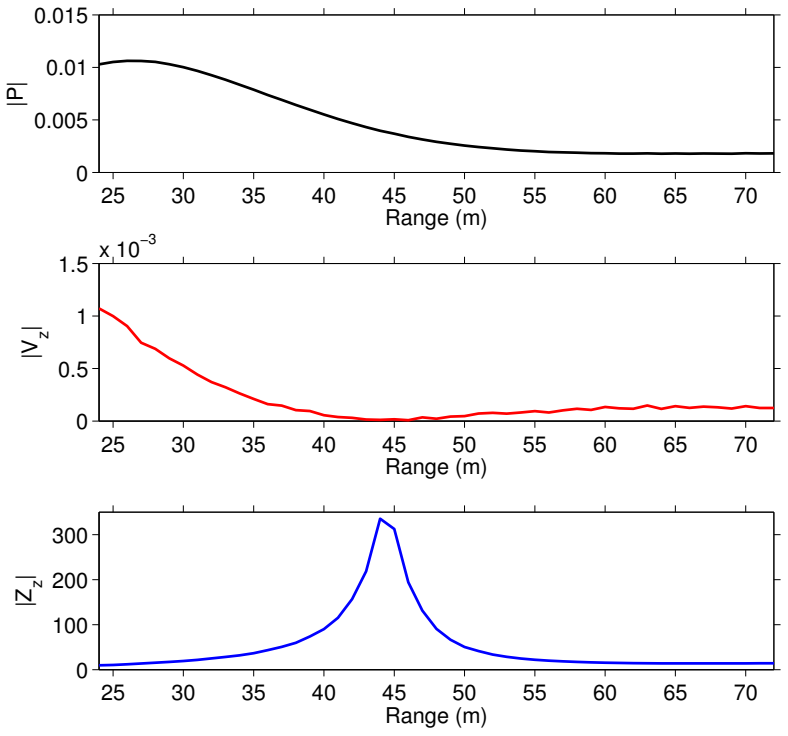

Figure 1: Predicted $P, V_{z}$ and $Z_{z}$ for the geoacoustic model of CANOGA 12 environmental model.

The UKF is used in paper due to its robustness for high nonlinear problems, which uses an unscented transform to first pick a minimal set of sample points around the mean and then propagates these samples through non-linear functions to recover the true mean and covariance of the estimate. Besides, the UKF does not require the calculation of Jacobians, which are particular difficult for complex sound propagation model and need to be calculated numerically as in extended Kalman filter [15] that may give poor performance [10].

\section{NUMERICAL SIMULATIONS FOR GEOACOUSTIC INVERSION}

Numerical simulations were conducted for environmental model of a mud environment offshore at the mouth of the Amazon river in Brazil (CANOGA 12). Based on prior information, the environmental parameters of the experimental area are given in Tab. I. Figure 1 is the predicted $P, V_{z}$ and $Z_{z}$ for the CANOGA 12 geoacoustic model for a sound frequency of $150 \mathrm{~Hz}$, with source $\left(d_{s}\right)$ and receiver $\left(d_{r}\right)$ depths set as $0.8 \mathrm{~m}$ and $4.0 \mathrm{~m}$, respectively. Their range variations are dependent on the waveguide and are observed here by the sequential UKF for environmental characterization.

For the preliminary simulations, acoustic data of six frequencies $(125 \mathrm{~Hz}, 150 \mathrm{~Hz}, 175 \mathrm{~Hz}, 200 \mathrm{~Hz}, 225 \mathrm{~Hz}$ and 250 $\mathrm{Hz})$ are used. The filter analyses acoustical data from $23 \mathrm{~m}$ to $73 \mathrm{~m}$ and with new data input for each $1 \mathrm{~m}$-increment. The bottom attenuation were not considered due to their mirror effects on the low-frequency sound fields in this very shallow waveguide. However, geometrical parameters of water depth $d_{w}$, the source $\left(d_{s}\right)$ and receiver $\left(d_{r}\right)$ depth are included.

In the test, synthetic $P, V_{z}$ and $Z_{z}$ data were first generated by the UKF simulator [16] with these parameters in Tab. I, and UKF estimator is then used to recover these parameters change along range. The initial state for the UKF estimator is slightly 

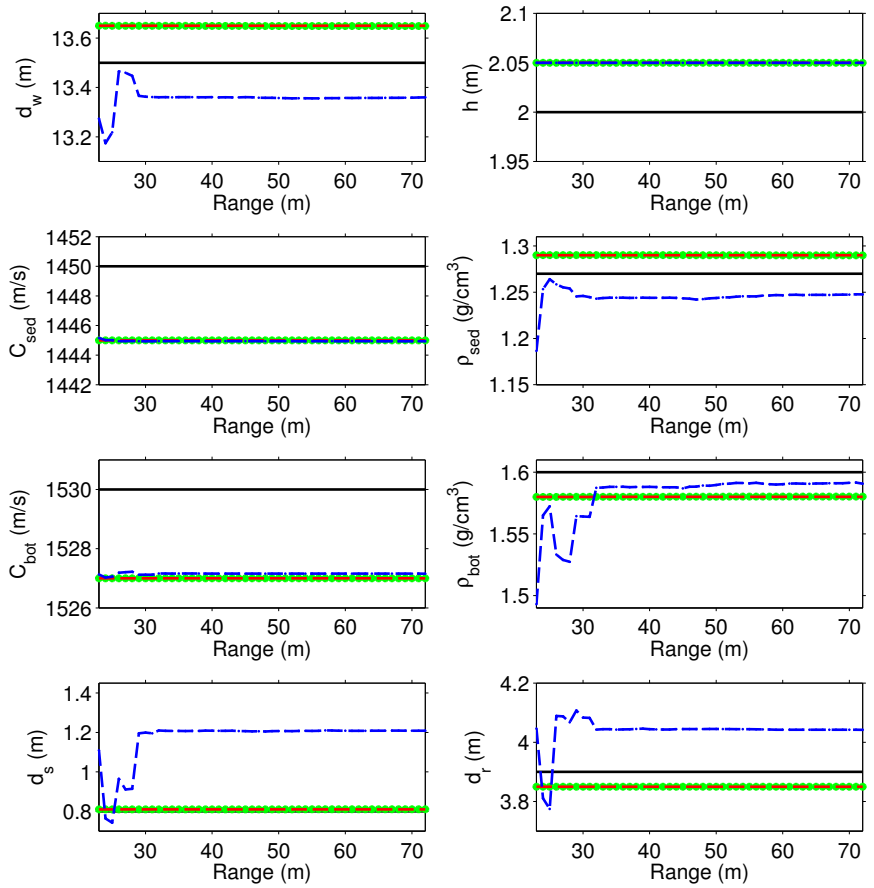

Figure 2: Tracked results for environmental parameters using $P, V_{z}$ and $Z_{z}$, black solid line is the true values for each parameter, green star are the result obtained from $P$, red dashed line for $V_{z}$ and blue dash-dotted line for $Z_{z}$.
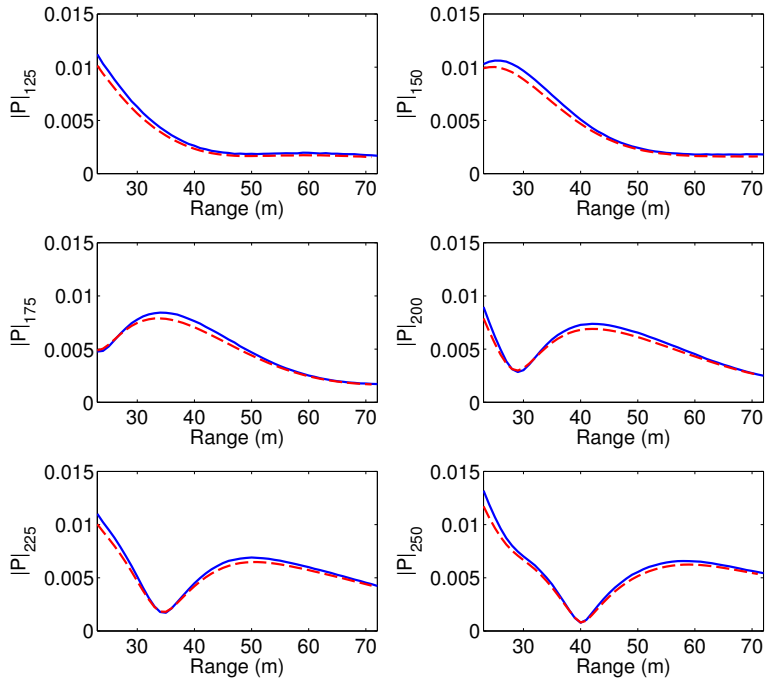

Figure 3: A comparison between the measurements (blue) and predictions by the UKF (red dashed line) for different frequency $P$.

different from that of simulator. The $R_{w w}$ and $R_{v v}$ are the same for $P, V_{z}$ and $Z_{z}$ data. For easiest case of estimating only one parameter and with other parameters fixed, the UKF can easily find their true values (not shown here), the results presented here are obtained using UKF to simultaneously estimate all these parameters.
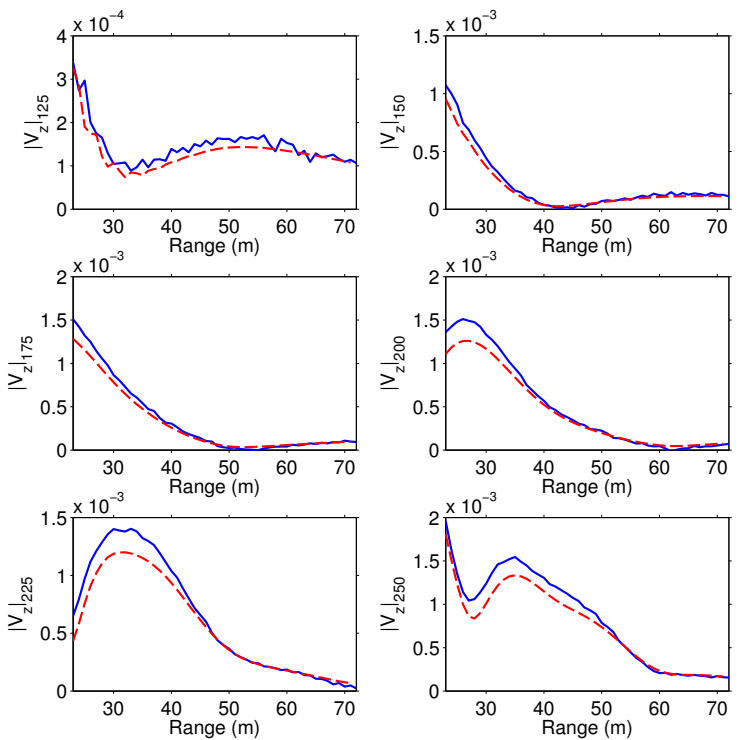

Figure 4: A comparison between the measurements (blue) and predictions by the UKF (red dashed line) for different frequency $V_{z}$.
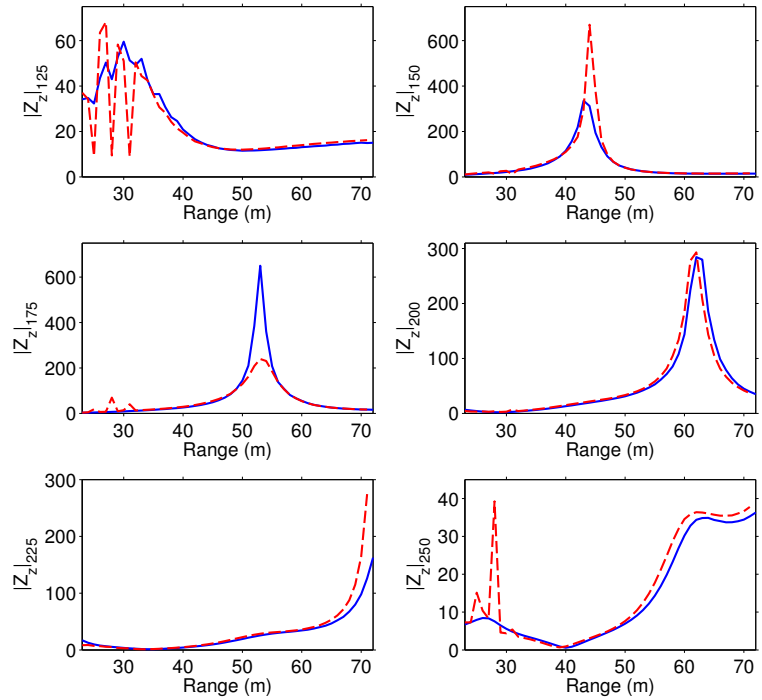

Figure 5: A comparison between the measurements (blue) and predictions by the UKF (red dashed line) for different frequency $Z_{z}$.

Figure 2 gives the results obtained from $P, V_{z}$ and $Z_{z}$ acoustic data. If only look at the Fig. 2, one cannot say the UKF was trying to estimate these parameters values, especially for the results from $P$ and $V_{z}$. However, the results from $Z_{z}$ show that the UKF was trying to find these parameter values, at least for $d_{w}, \rho_{s e d}, \rho_{b o t}$ and $d_{r}$ at the beginning. This may due to the very small covariance (1e-12) assigned for $R_{v v}$, so the UKF more believes previous estimates than the measurements and therefore only subtle corrections are made for current estimate.

Figures 3 and 4 give the comparisons between UKF pre- 
dictions and measurements for $P$ and $V_{z}$, respectively. The already good matches between predictions and measurement along range maybe give the reason why the UKF can not really update the estimates. However, when looking at Fig. 5, the UKF was trying to match measurements step by step before $35 \mathrm{~m}$, especially for the bottom densities which are typically insensitive parameters for traditionally MFP techniques. After $35 \mathrm{~m}$, the predictions and measurements agree well with each other and is believed the reason why the UKF stopped to converge as shown in Fig. 2. These results suggests more tests need to be conducted to test the effects of processing and measurement noise covariance matrix on the performance of UKF and the used for real ship noise data processing.

\section{CONClusion}

This paper introduced a sequential UKF for passive geoacoustic inversion using pressure and vector noise fields due to ship of opportunity. This filter analyses the range variations of different acoustical quantities for environmental characterization in shallow water. Numerical results demonstrated that the sequential filtering technique performs better when applied to $Z_{z}$ than that of $P$ and $V_{z}$ treated individually, especially for the bottom densities. In practice, ship data have complex time-varying spectral characteristics [17] that can introduce unexpected measurement noise to the UKF that can greatly degrade the accuracy of inversion results using $P$ or $V_{z}$ only. Due to the independent of the source spectral level for $Z_{z}$, it is preferred to be used for environmental characterization using the sound field generated from a ship of opportunity and expected to yield a more reliable inversion than that of $P$ or $V_{z}$.

\section{REFERENCES}

[1] C. B. Leslie. "Hydrophone for measuring particle velocity." J. Acoust. Soc. Am. , 28(4):711-715, 1956.
[2] H. S. Peng, and F. H, Li. "Geoacoustic inversion from vector hydrophone array in shallow water", Technical acoustics, 27(2):163-167, 2008.

[3] Q. Y. Ren, and J.-P. Hermand."Passive geoacoustic inversion using waveguide characteristic impedance: a sensitivity study," In Proc. eleventh Eur. Conf. on Underwater Acoustics (C. Capus, ed.), pp. 1394-1400, Institute of Acoustics, 2012.

[4] A. B. Baggeroer, W. A. Kuperman, and P. N. Mikhalevsky, "An overview of matched field methods in ocean acoustics," IEEE J. Ocean. Eng. 18, 401-424, 1993.

[5] A. Tolstoy, "Matched field processing for underwater acoustics," Singapore: World Scientific Pub., 1993.

[6] N. Chapman, S. Chin-Bing, D. King, and R. Evans, "Benchmarking geoacoustic inversion methods for range-dependent waveguides," IEEE J. Ocean. Eng., vol. 28, no. 3, pp. 320-330, Jul. 2003.

[7] M. D. Collins. "Nonlinear inversion for ocean-bottom properties." J. Acoust. Soc. Am., 92(5):2770-2783, 1992.

[8] S. M. Jesus. "Can Maximum Likelihood Estimators Improve Genetic Algorithm Search in Geoacoustic Inversion?" Journal of Computational Acoustics, vol. 6:73-82, 1998.

[9] J.-P. Hermand, "Broad-band geoacoustic inversion in shallow water from waveguide impulse response measurements on a single hydrophone: theory and experimental results," IEEE J. Ocean. Eng. vol. 24, no. 1, pp. 41-66, 1999.

[10] E. A. Wan, and R. Van Der Merwe, "Unscented Kalman Filter for nonlinear estimation", Adaptive Systems for Signal Processing, Communications, and Control Symposium 2000, 153-158, 2000.

[11] O. Carrière and J.-P. Hermand, "Sequential Bayesian geoacoustic inversion for mobile and compact source-receiver configuration," J. Acoust. Soc. Am. 131, 2668-2681, 2012.

[12] J.-P. Hermand and S. B. Vinzon, "CANOGA-CANALNORTE 12 cruise report," Federal University of Rio de Janeiro-Université libre de Bruxelles, Rio de Janeiro, Brazil, Tech. rep., 2012.

[13] L. M. Brekhovskikh and Yu. P. Lysanov. Fundamentals of Ocean Acoustics, volume 116. Springer, 3rd edition, 2003.

[14] R. E. Kalman, "A new approach to linear filtering and prediction problems," ASME J. Basic Eng. 82, 35-45, 1960.

[15] S. J. Julier and J. K. Uhlmann, "A new extension of the Kalman filter to nonlinear systems," Int. Symp. Aerospace/Defense Sensing, Simul. and Controls 3., 1997.

[16] James V. Candy, "Model-based signal processing," Wiley. com., 2005.

[17] P. T. Arveson and D. J. Vendittis, "Radiated noise characteristics of a modern cargo ship," J. Acoust. Soc. Am. 107, 118-129, 2000. 